\title{
New plasmonic materials in visible spectrum through electrical charging
}

Jiangrong Cao, Rajesh Balachandran, Manish Keswani, Krishna Muralidharan, Slimane Laref, et al.

Jiangrong Cao, Rajesh Balachandran, Manish Keswani, Krishna Muralidharan, Slimane Laref, Richard Ziolkowski, Keith Runge, Pierre Deymier, Srini Raghavan, Mamoru Miyawaki, "New plasmonic materials in visible spectrum through electrical charging," Proc. SPIE 8632, Photonic and Phononic Properties of Engineered Nanostructures III, 86321I (21 February 2013); doi: $10.1117 / 12.2002883$

SPIE. Event: SPIE OPTO, 2013, San Francisco, California, United States 


\title{
New plasmonic materials in visible spectrum through electrical charging
}

\author{
Jiangrong Cao ${ }^{\mathrm{a}}$, Rajesh Balachandran ${ }^{\mathrm{b}}$, Manish Keswani ${ }^{\mathrm{b}}$, Krishna Muralidharan ${ }^{\mathrm{b}}$, Slimane Laref ${ }^{\mathrm{b}}$, \\ Richard Ziolkowski ${ }^{\mathrm{c}}$, Keith Runge ${ }^{\mathrm{b}}$, Pierre Deymier ${ }^{\mathrm{b}}$, Srini Raghavan ${ }^{\mathrm{b}}$, and Mamoru Miyawaki ${ }^{\mathrm{a}}$ \\ ${ }^{a}$ Optics Research Laboratory, Canon U.S.A. Inc., 9030 S. Rita Road, Tucson, AZ 85747, USA \\ ${ }^{b}$ Department of Material Science and Engineering, University of Arizona, 1235 E. James E. Roger \\ Way, Tucson, AZ 85721, USA \\ 'Department of Electrical and Computer Engineering, 1230 E. Speedway Blvd, Tucson, AZ 85721, \\ USA
}

\begin{abstract}
Due to their negative permittivity, plasmonic materials have found increasing number of applications in advanced photonic devices and metamaterials, ranging from visible wavelength through microwave spectrum. In terms of intrinsic loss and permittivity dispersion, however, limitations on available plasmonic materials remain a serious bottleneck preventing practical applications of a few novel nano-photonic device and metamaterial concepts in visible and nearinfrared spectra.

To overcome this obstacle, efforts have been made and reported in literature to engineer new plasmonic materials exploring metal alloys, superconductors, graphene, and heavily doped oxide semiconductors. Though promising progress in heavily doped oxide semiconductors was shown in the near-infrared spectrum, there is still no clear path to engineer new plasmonic materials in the visible spectrum that can outperform existing choices noble metals, e.g. gold and silver, due to extremely high free electron density required for high frequency plasma response.

This study demonstrates a path to engineer new plasmonic materials in the visible spectrum by significantly altering the electronic properties in existing noble metals through high density charging/discharging and its associated strong local bias effects. A density functional theory model revealed that the optical properties of thin gold films (up to $7 \mathrm{~nm}$ thick) can be altered significantly in the visible, in terms of both plasma frequency (up to 12\%) and optical permittivity (more than 50\%). These corresponding effects were observed in our experiments on surface plasmon resonance of a gold film electrically charged via a high density double layer capacitor induced by a chemically non-reacting electrolyte.
\end{abstract}

Keywords: plasmonic metal, optical permittivity, electrochemistry double layer capacity

\section{INTRODUCTION}

As a class of optical materials, plasmonic metals have received growing interest in the optics and photonic community in the recent decade, due to their negative permittivity (real part) in visible and near infrared spectra. As more and more designs and applications in integrated photonics and metamaterials are proposed utilizing plasmonic metal constituents, the severe limitations of the few known plasmonic metals (e.g. Au and Ag) viable for visible and near infrared spectra become apparent, particularly in terms of excessive absorption loss and dispersion.

In this context, significant research efforts are underway to design (i) metals and metal-alloys with suitable electronic bandstructures [1], [2], (ii) superconductors, (iii) graphene nanostructures [3], and heavily doped oxides [4]. However, when it comes to negative permittivity (real part) in relatively high photon energy region (i.e. visible and infrared spectra), a viable alternative to the currently used plasmonic metals is still elusive [5], [6].

In this study, we have explored a new route to alter optical permittivity of a plasmonic metal, Au, by forming a high area density surface capacitor. Using both electronic structure modeling methods such as density functional theory (DFT) in conjunction with electrochemical methods examining high density surface capacitive properties of a Au-electrolyte interface, we have demonstrated the feasibility of engineering the optical permittivity of a plasmonic metal in the visible spectrum.

Photonic and Phononic Properties of Engineered Nanostructures III,

edited by Ali Adibi, Shawn-Yu Lin, Axel Scherer, Proc. of SPIE Vol. 8632, 86321I

(c) 2013 SPIE · CCC code: 0277-786X/13/\$18 - doi: 10.1117/12.2002883 


\section{A STRATEGY OF ALTERING PLASMONIC METAL'S PERMITTIVITY}

Just like any optical material (e.g. glasses, crystals, etc.), a plasmonic material's optical permittivity is determined by its electronic properties. Specifically, the optical permittivity of Au consists of two important electronic contributions. The first term describes the collective conduction electron plasma oscillation, i.e. the intraband term, which results in the characteristic permittivity dispersion frequently characterized by the Lorentz-Drude model. The second term is the undesired interband transition term, which contributes mainly to the absorption loss but not the negativity of the permittivity (real part). For $\mathrm{Au}$, the intraband term is described by an electron plasma frequency of $\sim 9.0 \mathrm{eV}$, and a collision frequency of $\sim 0.08 \mathrm{eV}[7]$.

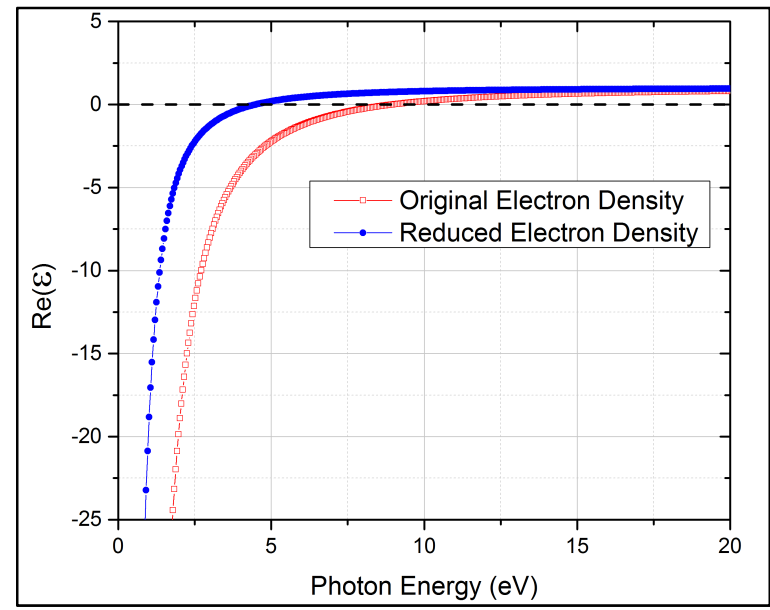

Figure 1: The real part of permittivity dispersion modeled by the Drude model for Au with plasma frequency $9.0 \mathrm{eV}$ (red squares), and for a hypothetical material with lower conduction electron density than Au (blue circles).

Using the Drude model for $\mathrm{Au}$, the red squares in Figure 1 illustrates how the real part of its permittivity crosses from a positive to a negative value, when the photon energy is reduced below $9.0 \mathrm{eV}$. Here, we first concentrate on the intraband term, while omitting the interband absorption term.

Hypothetically, if one can reduce the free electron density in solid state Au by a factor of 4, then the resulting metal would have a permittivity dispersion as represented by the blue circles in Figure 1, which marks a significant change for an optical material functioning in the visible wavelength photon energy span (i.e. between $1.6 \mathrm{eV}$ and $3.1 \mathrm{eV}$ ). The same kind of the permittivity change could also be achieved by a combination of reduced density and increased effective mass for the conduction electrons.

Moreover, if one can modulate the electron density in solid state Au even by a fraction of one electron per Au atom, then it should significantly impact the metal's optical permittivity by altering the following four properties simultaneously as enumerated below:

- the conduction electron density, thereby directly influencing the plasma frequency.

- the effective mass of electrons, which influences the plasma frequency too.

- the collision frequency in the intraband Drude term.

- the electronic bandstructure, which could modify the interband transition term.

To achieve the ability to modulate the effective charge density within solid-state $\mathrm{Au}$, one should note that typical semiconductor techniques may not be suitable, given the large free carrier density (at $10^{23} / \mathrm{cm}^{3}$ ). However, the rapid advancement in nano-plasmonic and metamaterial designs has resulted in the thickness of plasmonic metal components to decrease to be below $10 \mathrm{~nm}$ [8] and approaching $5 \mathrm{~nm}$. Thus modulating the surface charge on nanometric thin metal 
films would be sufficient to achieve significant optical modulation for most of those leading edge nano-plasmonic and metamaterial designs.

While the focus of this work is to provide experimental evidence for charge-density modulation and hence altering the thin-film metals' permittivity, it is worthwhile to examine the DFT-based study we initiated. Specifically, our simulation results revealed that thin gold films' (up to $7 \mathrm{~nm}$ thick) optical property in the visible spectrum can be altered significantly, in terms of both plasma frequency (up to $12 \%$ ) and optical permittivity (more than $50 \%$ ). These data is reported in a dedicated article [9].

Experimentally, altering the electron density in nanometric Au thin films is possible either by applying a strong bias in vacuum [10], or by forming a high density capacitor at one (or both) surface of the Au slab, which can displace high density surface charge with only low voltage biasing [11]. In particular, for a surface capacity of $100 \mu \mathrm{F} / \mathrm{cm}^{2}$ or larger, we expect the electron density in the Au slab can be altered significantly with only few volts of electrical bias applied. A straight-forward way to achieve this is by placing the nanometric Au film inside an electrolyte solution containing smallsized ions (e.g. F, $\mathrm{Cl}, \ldots$ ), and suitably biasing the Au film with respect to the liquid. This can enable the formation of a capacitive charging layer at the Au-electrolyte interface with equal and opposite charge displacement within the electrolyte countered by an equivalent electron displacement within the first nanometer or so inside the Au film as , as discussed below.

\section{EXPERIMENTAL METHOD}

To create and to optically probe the optical permittivity change of an Au thin film, a setup combining electrochemistry and optical attenuated total reflection was utilized. An illustration of the overall measurement system is shown in Figure 2 .

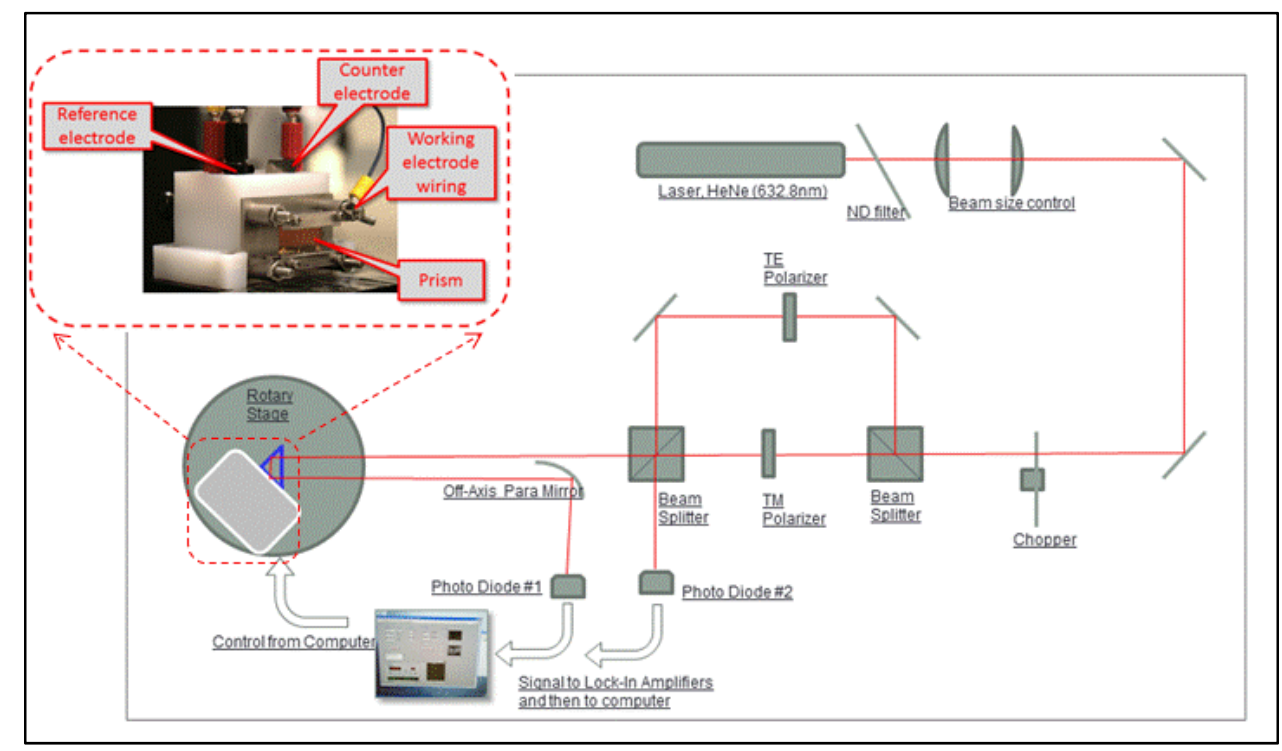

Figure 2: An illustration of the experimental setup used in this study. It combines an electrochemical static charging setting and a retro-reflecting optical attenuated reflectance setting. The inset (top-left) is a photograph of the measurement sample unit mounted on the rotary stage.

Electrochemically, the UHMWPE (Ultra-high-molecular-weight polyethylene) cell was machined to contain $40 \mathrm{ml}$ of $0.5 \mathrm{M} \mathrm{KCl}$ electrolyte solution, and has a circular $1-\mathrm{cm}^{2}$ sidewall window opening sealed by an Au coated facet of a right angle prism, which is formed by NSF-11 high index glass. The Au coating was produced through evaporation of $5 \mathrm{~nm}$ Ti and $50 \mathrm{~nm}$ Au onto a facet of the NSF-11 glass prism in a commercial high vacuum e-beam evaporator (Edwards Auto 306) at $0.03 \mathrm{~nm} / \mathrm{sec}$ deposition speed. During the experiments, the Au film was electrically connected to the working electrode of a potentiostat, and it was statically biased against a Pt reference electrode in the same $\mathrm{KCl}$ solution. 


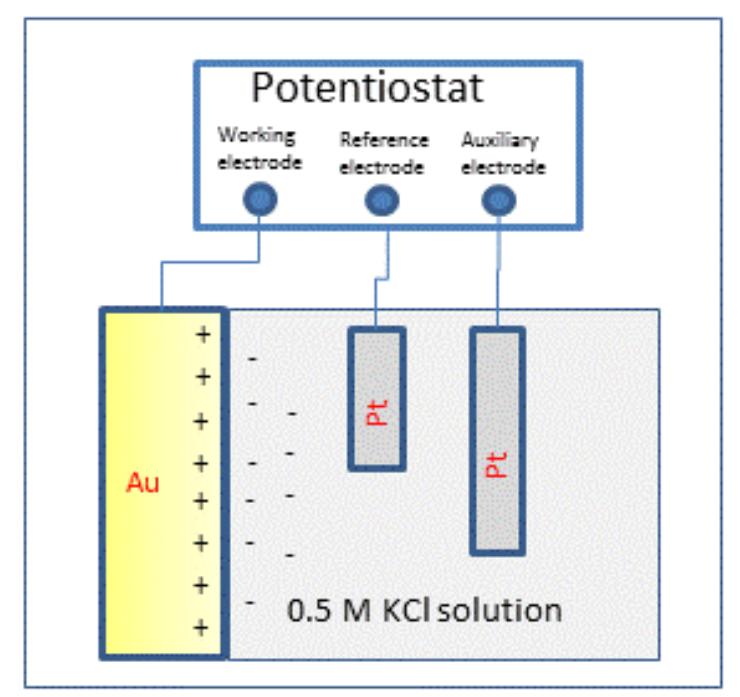

Figure 3: An illustration showing how electrically biasing a Au film against a Pt reference electrode in a $\mathrm{KCl}$ solution forms a statically positive charging layer just below the Au surface.

Optically, a collimated TM (transverse magnetic with respected to the Au film) polarized laser beam (HeNe 633nm) incidents on the glass prism. The laser beam then experienced two reflections (one at uncoated prism facet, and one at the Au coated facet) and retro-reflected parallel with the incident beam. This retro-reflected beam is then focused by an off-axis parabolic mirror to a photo detector (i.e. photo diode \#1 in Figure 2). The ratio between two photo detectors (c.f. Figure 2) as a function of rotation angle of the rotary stage (c.f. Figure 2) then became the optical signal we measured.

\section{EXPERIMENTAL RESULTS}

The Au film electrical charging condition was characterized by cyclic voltammetry measurements. Figure 4 plots the results of three cyclic voltammetry scans with progressively increasing bias windows. The different hysteresis of the thee scan-results in Figure 4 shows the solid state Au starts to be oxidized by $\mathrm{Cl}^{-}$anions when bias is higher than $0.15 \mathrm{~V}$, the dissolved oxygen gas in the solution starts to be reduced when bias is lower than $-0.35 \mathrm{~V}$, and by staying within the bias window between $-0.3 \mathrm{~V}$ and $+0.1 \mathrm{~V}$ the Au film surface only experiences surface charge and discharge without chemical reaction. This is the bias window we worked within, during the following optical measurements. A time integration of the cyclic voltammetry curve in Figure 4 gives an estimation of charging of Au film surface at the density as high as 0.2 electrons per surface Au atom. 


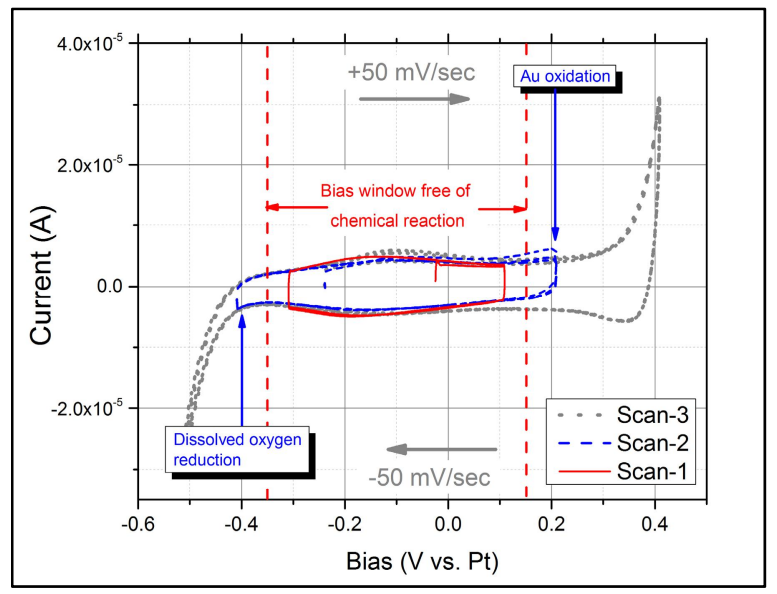

Figure 4: Experimentally measured cyclic voltammetry responses from the Au film coated on the glass prism. A Pt reference electrode was used. The potential was first ramped towards the positive direction (i.e. anodic direction). Three scans with progressively increasing bias windows were performed.

The attenuated total reflection for TM polarized light was measured with various conditions in the electrochemical cell. And the results are plotted in Figure 5.

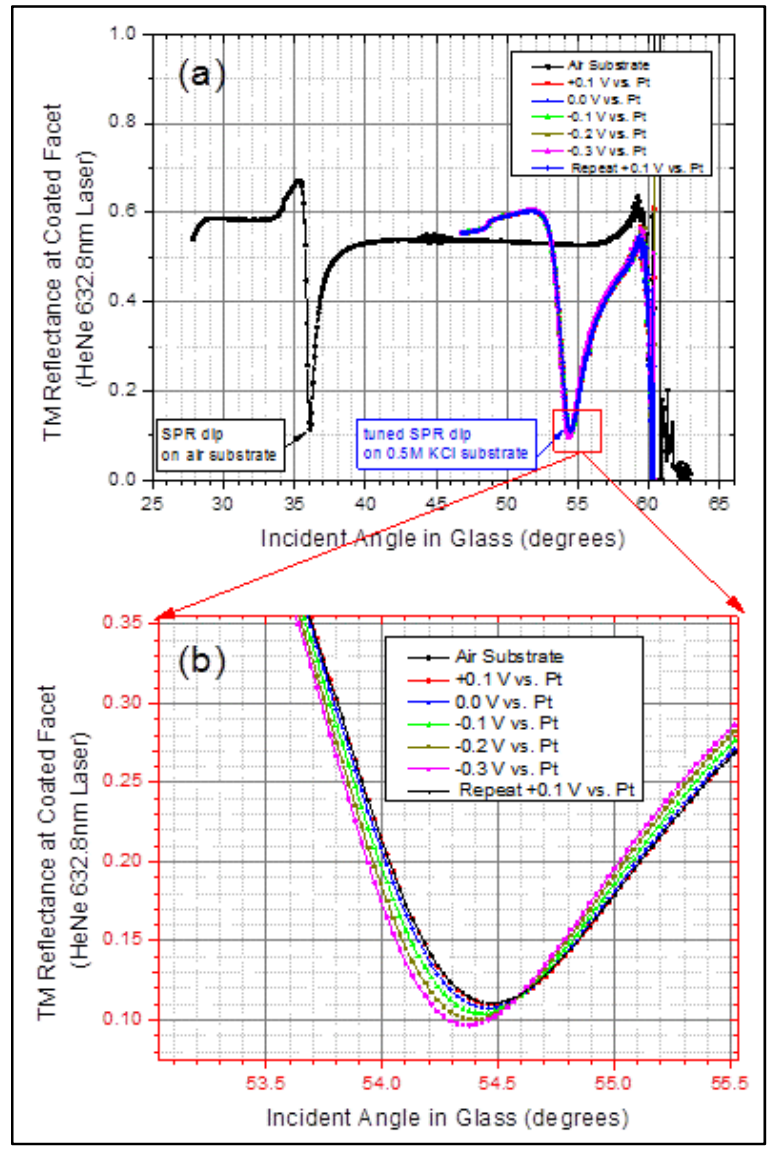

Figure 5: Experimentally measured attenuated total reflection of the Au coated prism facet with various conditions in the electrochemical cell, in a wide angle scale (a) and in a zoom-in scale (b) showing the varying the Au film bias potential is modifying electronic structure of the Au surface layer, which leads to optical permittivity changes impacting the TM reflectance curves. 
Then, relying on the high accuracy of the absolute reflectance and incident angle measurement achieved in our experiments, the attenuated total reflection curves were translated into the $\mathrm{Au} / \mathrm{Ti}$ film thickness and permittivity information through a transfer matrix based inverse solver. Figure 6 shows how well our inverse solver with multilayer model fits the experimental data and how precisely they match.

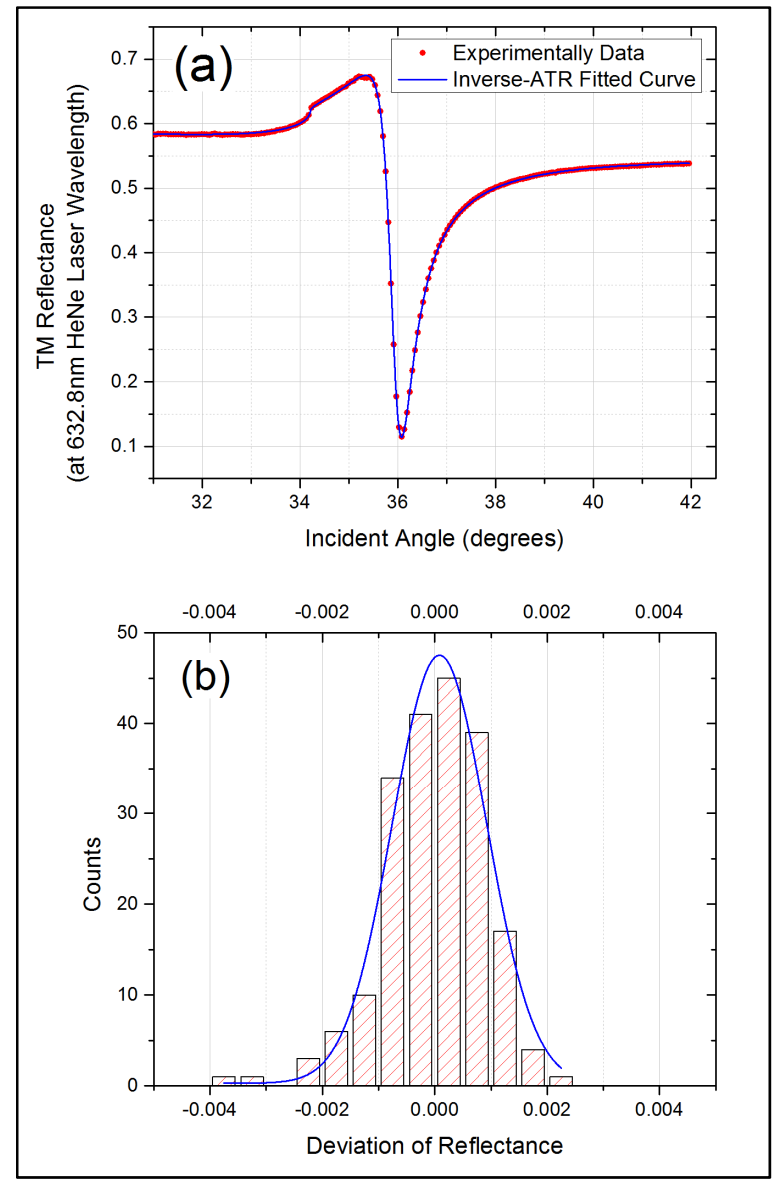

Figure 6: (a) is an experimental attenuated total reflection profile (red dots) was fitted with a Ti/Au two layer model through an inverse solver (blue line). (b) is a histogram showing the deviation between the two curves in (a) is a normal distribution with a narrow width of 0.002 , where the blue envelope curve is a Gaussian fit to the histogram.

Then, the permittivity of an Au surface layer $(0.5 \mathrm{~nm}$ below film-electrolyte interface) was extracted by feeding the experimental data in Figure 5 into the inverse solver. As shown in Figure 7, the extracted permittivity of this electrically charge Au layer has two most notable characteristics:

- The real part of the in-plane permittivity (i.e. dominant permittivity component forming the surface plasmon resonant) increases significantly from its literature value (i.e. -12.0 [7]), as the Au surface become more positively charged at higher bias potential.

- The out-of-plane permittivity shows clear anisotropy against the in-plane components. This anisotropy arises from the quantum confinement effect in this $0.5 \mathrm{~nm}$ thickness direction of the charged surface layer, which perturbs the electronic state in the out-of-plane direction further than the in-plane direction. 


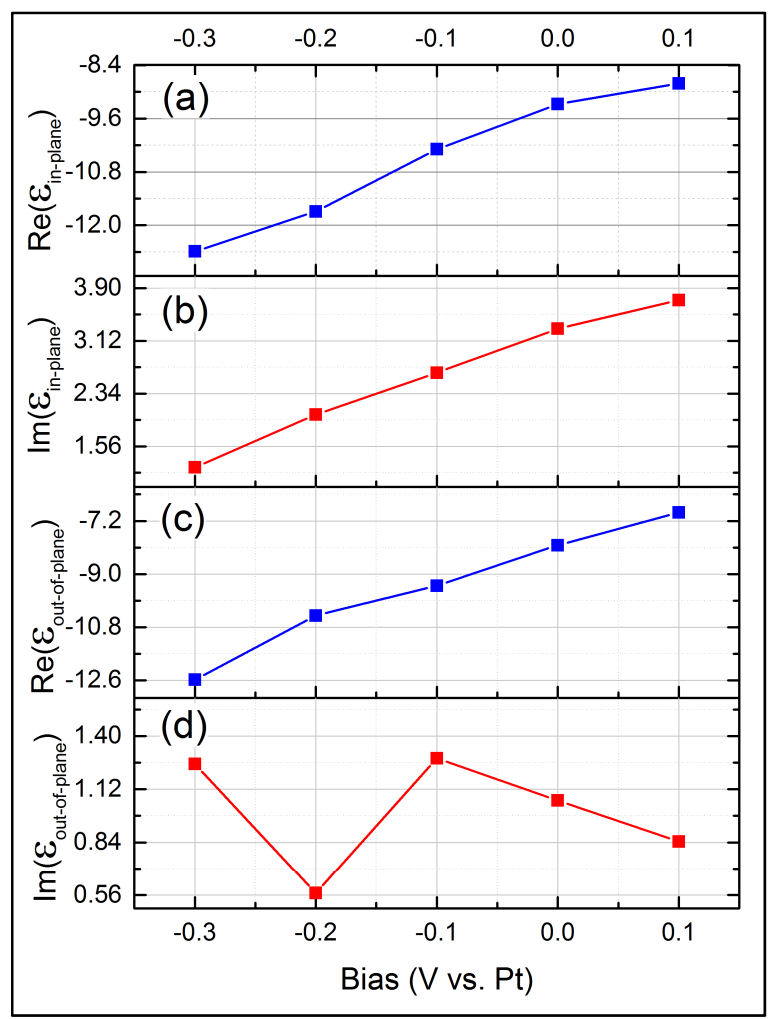

Figure 7: the extracted anisotropic permittivity of a $0.5 \mathrm{~nm}$ electrically charged layer just below the Au surface. It has two components, where the in-plane direction refers to in the plane of the Au film, and the out-of-plane direction refers to the thickness direction of the Au film.

\section{SUMMARY}

We have outlined a path to engineer plasmonic material thin films' optical permittivity in visible spectrum by significantly altering the electronic properties of thin-film noble metals through high density charging/discharging. Using only $0.4 \mathrm{~V}$ electrical bias, we have experimentally demonstrated changing a $0.5 \mathrm{~nm}$ Au layer's permittivity from 12.6 to about -9.0 at $633 \mathrm{~nm}$ wavelength, which is resulted from a high density surface capacitive charge accumulation.

\section{REFERENCES}

[1] Blaber, M. G., Matthew D. A., and Ford, M. J., "Search for the Ideal Plasmonic Nanoshell: The Effects of Surface Scattering and Alternatives to Gold and Silver," J. Phys. Chem. C. 113(8), 3041-3045 (2009).

[2] Khurgin, J.B., and Sun, G., "In search of the elusive lossless metal," Appl. Phys. Lett. 96, 181102 (2010).

[3] Vakil, A. and Engheta, N., "Transformation Optics Using Graphene," Science 332, 1291 (2011).

[4] Naiki, G. V., et al., "Demonstration of $\mathrm{Al}: \mathrm{ZnO}$ as a plasmonic component for near-infrared metamaterials," PNAS 109(23), 8834-8838 (2012).

[5] Boltasseva, A. and Atwater, H.A., "Low-Loss Plasmonic Metamterials," Science 331, 290 (2011).

[6] Tassin, P., et al., "A comparison of graphene, superconductors and metals as conductors for metamaterials and plasmonics," Nature Photonics 6, 259-264 (2012).

[7] Johnson, P.B., and Christy, R.W., "Optical constants of the noble metals," Phys. Rev. B. 6, 4370-4379 (1972)

[8] Krishnamoorthy, H.N.S., et al., "Topological Transitions in Metamaterials," Science 336, 205 (2012).

[9] Slimane, L., et al., "Size-dependent permittivity and intrinsic optical anisotropy of nanometric gold thin films: a density functional theory study," submitted to Optics Express (2012)

[10] Lapsley, M. I., et al., "Shifts in plasmon resonance due to charging of a nanodisk array in argon plasma," Appl. Phys. Lett. 100, 101903 (2012).

[11] Shao, L.H., et al., "On the mechanism of electrochemical modulation of plasmonic resonances," Appl. Phys. Lett. 101, 121109 (2012). 